\title{
EL GRADIENTE SOCIAL DE LA OBESIDAD EN ESPAÑA. ¿QUÉ SABEMOS Y QUÉ DEBERÍAMOS SABER? ${ }^{1}$
}

\author{
Autoras: Josefina Panetta ${ }^{2}$ \\ Servicio Canario de Salud, Fuerteventura \\ Beatriz G. López-Valcárcel ${ }^{3}$ \\ Universidad de Las Palmas de Gran Canaria \\ Departamento de Métodos Cuantitativos en Economía y Gestión \\ Campus de Tafira, Las Palmas de Gran Canaria
}

\section{Resumen}

Introducción: la OMS define el gradiente social como la desigualdad en salud a lo largo del continuo de la condición socioeconómica. La obesidad es resultante de múltiples y complejas causas y refleja un problema de desigualdad social. El

\footnotetext{
${ }^{1}$ Este trabajo fue financiado por el Plan Nacional de I+D+I de España, Investigación Fundamental Orientada a los Retos de la Sociedad, Proyecto ECO2013-48217-C2-1-R.

${ }^{2}$ jpanettamonea@gmail.com

${ }^{3}$ beatriz.lopezvalcarcel@ulpgc.es
} 
objetivo de este artículo es revisar y discutir la evidencia científica disponible sobre la asociación entre situación socioeconómica (renta, ocupación, clase social y educación) y sobrepeso/obesidad en España en niños y adultos.

Metodología: revisión crítica de la literatura en PubMed y Econlit de estudios publicados entre 2000 y 2016 que miden el gradiente socioeconómico de la obesidad, en niños y adultos.

Resultados: se han revisado 17 estudios primarios que miden el gradiente socioeconómico de la obesidad en España y otros 8 que comparan España con otros países. Los mecanismos que explican el gradiente son múltiples y abarcan la menor receptividad a mensajes y recomendaciones de prevención primaria, patrones de alimentación menos saludable entre niños y adolescentes de condición socioeconómica baja y el entorno obesogénico o de bajo nivel socioeconómico. Para medir el gradiente se utilizan el Îndice de Desigualdad Absoluta, el Índice de Desigualdad Relativa, el Índice Concentración y el porcentaje atribuible al nivel socioeconómico desfavorable. Aunque la prevalencia global de la obesidad en adultos parece que se está estabilizando en España, hay un fuerte gradiente social inverso por nivel educativo y por situación socioeconómica baja tanto en adultos como en niños. La educación es el factor de desigualdad de mayor impacto en cualquier grupo etario. Las niñas y mujeres obesas sufren una doble discriminación, pues el gradiente es significativamente mayor que para el género masculino.

Conclusiones: son necesarios más estudios con alta calidad metodológica con diseño prospectivo a largo plazo y medición objetiva del índice de masa corporal para monitorizar tendencias y contribuir al diseño de políticas efectivas contra la obesidad, que no solamente son políticas de salud sino también de equidad.

Palabras clave: obesidad; factores socioeconómicos; desigualdad en salud; gradiente social.

\title{
The social gradient of obesity in Spain. What do we know and what should we know?
}

\begin{abstract}
Introduction: WHO defines the social gradient as inequality in health along the continuum of socioeconomic status. Obesity is the result of multiple and complex causes and reflects a problem of social inequality. The objective of this article is to review and discuss available scientific evidence on the association between socioeconomic status (income, occupation, social class and education) and overweight / obesity in Spain in children and adults.
\end{abstract}


Methodology: Critical review of the literature through PubMed and Econlit. Studies measuring the socioeconomic gradient of obesity in children and adults, published between 2000 and 2016.

Results: 17 primary studies measuring the socioeconomic gradient of obesity in Spain have been reviewed, and another 8 that compare Spain with other countries. The mechanisms that explain the gradient are multiple and include less receptivity to messages and recommendations of primary prevention, less healthy eating patterns among children and adolescents of low socioeconomic status, and the obesogenic environment of low socioeconomic level households. To measure the gradient, the Absolute Inequality Index, the Relative Inequality index, the Concentration Index and the percentage attributable to the unfavorable socioeconomic level are used. Although the overall prevalence of obesity in adults seems to be stabilizing in Spain, there is a strong inverse social gradient by educational level and low socioeconomic status in both adults and children. Education is the most important inequality factor in any age group. Obese girls and women suffer from double discrimination, as the gradient is significantly higher for them than for the male gender.

Conclusions: more studies are needed with high methodological quality and a long-term prospective design and objective measurement of body mass index to monitor trends and contribute to the design of effective policies against obesity, which are not only health policies but also policies enhancing equity.

Key words: obesity; socioeconomic factors; health inequality; social gradient.

\section{INTRODUCCIÓN CONCEPTUAL. EL GRADIENTE Y SUS ORÍGE- NES. OBJETIVOS DEL ARTÍCULO}

En este artículo se revisa la evidencia disponible sobre las desigualdades sociales relacionadas con la obesidad en España, y se detectan las carencias de información más relevantes. Pudiera parecer extraña la presencia de este tema en una revista multidisciplinar con un enfoque económico-empresarial, pero además de un problema de salud, la obesidad impone un coste social cuantioso y pérdidas de productividad de los trabajadores. El gradiente social de la obesidad se refleja también en la discriminación y estigma de los trabajadores obesos, lo cual entronca con la teoría económica de la discriminación. Por otra parte, la importancia económica de los sectores y las empresas relacionados con la alimentación y con el ejercicio físico es cada vez mayor. 
La obesidad es un factor de riesgo para la salud resultante de causas múltiples y complejas y susceptible de interacciones sociales que imprimen dinámica en los territorios (Christakis y Fowler, 2007). Entre las causas proximales se señalan los cambios en el estilo de vida caracterizados por la reducción de la actividad física y una mayor ingesta de comida rápida, sabrosa, barata y altamente energética, así como la promoción y persuasión de este tipo de comidas (Sassi y OECD, 2010). Los determinantes de la obesidad se dan a tres niveles interrelacionados: un nivel macro, que incluye factores sociales, políticos e históricos, que afectarían a la disponibilidad y calidad de los condicionantes de vida, y a las consecuencias de las políticas públicas (distribución de los recursos, composición racial, aplicación de la ley, valor de las viviendas, calidad de las escuelas); un nivel meso que afectaría al barrio y sus condiciones de vida, como la reputación, la disponibilidad y calidad de los servicios, su atractivo en general; y a un nivel individual, en el que operan factores como la predisposición genética, la clase social, las tradiciones culturales y las actitudes. Cada uno de esos tres niveles tiene influencias directas e indirectas sobre las opciones de comportamiento dietético y de actividad física, que pueden en última instancia afectar al peso corporal y a la morbilidad relacionada con él.

Ese conjunto de causas complejas y dinámicas da lugar a una distribución desigual del problema de la obesidad según posición socioeconómica, y se refleja en un determinado patrón geográfico de la obesidad -nivel de urbanización, nivel económico de la zona-.

Hay dos preguntas relevantes, una se refiere al nivel (prevalencia de obesidad y sobrepeso) y la otra a su distribución interpersonal. Ambas preguntas son susceptibles de análisis estático y dinámico (tendencias), y pueden formularse para grupos específicos de población -niños, adolescentes, adultos, adultos mayores-y por género.

El gradiente social es la desigualdad (injusta) en salud a lo largo del continuo de la condición socioeconómica: "Gradiente social en salud significa que las desigualdades de salud afectan a todos" (Marmot et al., 2008).

Uno de los ejes principales del debate sobre los determinantes sociales de la obesidad es la influencia relativa de los determinantes individuales y del entorno. La educación individual contribuye a la determinación de la posición socioeconómica de la persona, que a su vez tiene influencia en la probabilidad de ser obeso (Devaux y Sassi, 2013). La educación aumenta el conocimiento y por tanto la capacidad de seleccionar una dieta saludable y de hacer un uso eficiente de la atención médica. Pero la educación de los miembros del hogar podría tener una influencia directa sobre la probabilidad individual de ser obeso, por un efecto concurrente con la educación individual. En el modelo acumulativo de Campbell (Devaux et al., 2011), el impacto de la educación individual en la obesidad es consistente y aditivo con el impacto de la educación de otros miembros de la misma comunidad. Por tanto, la probabilidad de que un individuo pueda convertirse 
en obeso dependerá tanto del nivel de educación individual como de la educación de los otros miembros de la comunidad.

Por otra parte, la renta y la clase social, basada en la ocupación del cabeza de familia, son determinantes socioeconómicos que afectan a la familia entera, no solamente al individuo, por lo que la obesidad debe ser analizada a nivel de individuos y de hogares. Los ingresos del hogar pueden influir en el estilo de vida y en el acceso a los recursos, a menos ingresos mayor riesgo de obesidad, dada la estructura de precios relativos de los alimentos (Knai et al., 2012). Además, las familias comparten entorno, y la literatura señala efectos del entorno obesogénico o saludable sobre la obesidad (Black y Macinko, 2008).

Algunos autores explican el gradiente social de la obesidad mediante el paradigma del estrés. Las personas de baja posición socioeconómica que viven en barrios pobres tienen pocas opciones de mejorar, deben hacer frente a la marginación, discriminación, aislamiento y falta de control sobre su vida. Comer de forma compulsiva y fumar representan formas de placer y relajación, y la capacidad de renuncia a ellas es limitada. En la literatura de la economía de la salud se ofrece como ulterior argumento para explicar el gradiente que las personas de posición socioeconómica baja, al tener menor esperanza de vida y mayor riesgo de muerte prematura, tienen menos razones para invertir en el futuro y más para centrarse en el presente. Su tasa de descuento intertemporal es más alta que la de las personas en posición socioeconómica alta. En suma, los mecanismos y procesos que argumentan las diferencias en estilos de vida entre grupos socioeconómicos son diversos y posiblemente complementarios (Pampel, Krueger y Denney, 2010).

Se proponen soluciones (Lobstein, Millstone y PorGrow Research, 2007; Magnusson, 2014) fundamentadas en los modelos conceptuales que explican las "causas de las causas", o causas distales del aumento de la prevalencia de la obesidad y de su gradiente social: los modelos genético, de transición económica, de convergencia global, de la distribucion de alimentos y cambios tecnologicos, de la transición cultural, de la transición nutricional, y el modelo psicosocial aportan causas y soluciones diversas, y complementarias, para combatir la epidemia (Sassi y OECD, 2010).

Los gobiernos de los países desarrollados han abordado el problema de la obesidad con diversos instrumentos y políticas, a través de intervenciones individuales, comunitarias y sociales (Hillier-Brown et al., 2014), con regulaciones y medidas fiscales sobre alimentos no saludables y mediante intervenciones basadas en el conocimiento de la Economía del Comportamiento y el nudging (Sassi y OECD, 2010). Por otra parte, en orden a diseñar políticas adecuadas es importante conocer la influencia de las diferencias absolutas y de las relativas en el nivel socioeconómico sobre la incidencia de sobrepeso y obesidad. Una corriente de la literatura de salud pública sugiere que haciendo desaparecer las diferencias en educación mediante un "scaling-up" generalizado se resolvería el gradiente. 
Sin embargo, la efectividad de estas hipotéticas intervenciones podría no ser la esperada porque a gran escala no necesariamente se reproducirían los impactos o efectos conseguidos a pequeña escala (Banerjee y Duflo, 2009).

Este artículo revisa y discute la evidencia científica disponible sobre la asociación entre situación socio-económica (renta, ocupación, clase social y educación) y sobrepeso/obesidad en España en niños y adultos, así como la cuantificación empírica del gradiente social en la obesidad, entendido como una medida sintética de la distribución del problema de la obesidad a lo largo de toda la distribución poblacional del nivel socioeconómico. "Education, income, and occupational class cannot be used interchangeably as indicators of a hypothetical latent social dimension. Although correlated, they measure different phenomena and tap into different causal mechanisms" (Geyer et al., 2006). El texto se basa en una revisión crítica de la literatura científica publicada desde 2000. No ha sido posible realizar un meta-análisis porque no todos los estudios cuantifican los efectos con las mismas medidas y por la heterogeneidad de diseños y poblaciones en estudio.

\section{REVISIÓN DE LA LITERATURA: CARACTERÍSTICAS DE LOS ES. TUDIOS EMPÍRICOS SOBRE EL GRADIENTE PARA ESPAÑA}

Hemos revisado 17 estudios empíricos originales que miden el gradiente socioeconómico de la obesidad en España, y otros 8 que comparan el gradiente en España con otros países. Se publicaron entre 2000 y 2016. La Tabla 1 resume sus principales características. Entre los estudios para España, la mayor parte (12/17) utilizan datos transversales, o bases de datos transversales independientes en distintos momentos de tiempo (4/17). Únicamente uno analiza datos longitudinales, siguiendo a una cohorte durante 12 años (Cirera et al., 2015). La mayor parte (12/17) utilizan datos procedentes de encuestas ad-hoc o registros específicos, también 12 de los 17 miden objetivamente el IMC, mientras que en cinco casos el individuo lo autorreporta. Más o menos se reparten igualitariamente entre estudios para adultos (8/17) y para niños, adolescentes y/o jóvenes (9/17). Tres de los 8 estudios de adultos se concentran en la población mayor de 60 años.

El método estadístico más empleado es la regresión logística multivariante (7/17), y la variable dependiente es obesidad y/o sobrepeso en 12 de los 17 trabajos. Los tamaños muestrales oscilan entre unos pocos cientos de sujetos (Garcinuno et al., 2010) y casi dos cientos mil niños, con datos de un registro escolar en Aragón (Moreno et al., 2004).

Los estudios internacionales revisados, que incluyen a España, frecuentemente tienen por objetivo la comparación de prevalencia de obesidad y/o sobrepeso entre países y en el tiempo, además de medir el gradiente. 


\section{RESULTADOS}

\subsection{Indicadores antropométricos y diagnóstico sobrepeso/obesidad}

La mayor parte de los trabajos se basan en el Índice de Masa Corporal (IMC), que se calcula como el peso corporal (en kilogramos) dividido por la estatura (metros) al cuadrado $\left(\mathrm{kg} / \mathrm{m}^{2}\right)$. Para definir el estado nutricional se emplean diferentes estándares de referencia del IMC (OMS, IOTF y tablas de crecimiento CDC). Para los adultos se suele utilizar el estandar OMS (se clasifica de bajo peso cuando el IMC es $<18 \mathrm{~kg} / \mathrm{m}^{2}$, normal entre $18,5-24,99 \mathrm{~kg} / \mathrm{m}^{2}$, sobrepeso entre 25 y $29,99 \mathrm{~kg} / \mathrm{m}^{2}$ y obesidad cuando es $\geq 30 \mathrm{~kg} / \mathrm{m}^{2}$ ).

Para niños y adolescentes hay menos consenso respecto a la medida más adecuada de sobrepeso y obesidad (Sweeting, 2007). Uno de los estudios de referencia para España, el enKid (Serra-Majem et al., 2003), define obesidad a partir de los valores del índice de masa corporal iguales o superiores a los valores de los percentiles 97 y 85, respectivamente, de las tablas de referencia españolas de Hernández et al de 1988. Otra referencia para España es el estudio AVENA, de obesidad en adolescentes (Jimenez-Pavon et al., 2010) que además del IMC con referencia a unos estándares definidos, obtiene otras muchas medidas de sobrepeso y obesidad infanto-juvenil. Otros estudios (Costa-Font y Gil, 2013; DuarteSalles et al., 2011) utilizan los mismos percentiles ya citados (97 y 85) pero con valores de referencia alternativos, como los de la Fundación Orbegozo (Sobradi1lo, Aguirre y Aresti, 2004).

La opción más frecuente en los estudios para la infancia (Garcinuno et al., 2010; Moreno et al., 2004; Gonzalez Jiménez et al., 2012; Larranaga et al., 2007) es el uso de los estándares del Grupo Internacional de Trabajo sobre Obesidad (IOTF) también de la OMS, que vincula las definiciones de sobrepeso/obesidad en el adulto con los percentiles en niños, con la finalidad de establecer puntos de corte pediátricos para edades entre 3-20 años (valores $\geq$ percentil 85 , < percentil 95 define riesgo de sobrepeso; valor e $\geq$ percentil 95 define obesidad, que se correlaciona con un $\mathrm{IMC}>30 \mathrm{~kg} / \mathrm{m}^{2}$ ) a partir de datos internacionales de poblaciones infantiles (Cole et al., 2000). Estos estándares internacionales se utilizan también en España para definir obesidad y sobrepeso.

\subsection{Indicadores de posición socioeconómica}

En cuanto a la fuente del gradiente, es muy llamativo que la gran mayoría de los estudios consideran la educación (14/17); 10 de los 17 analizan el gradiente de clase social u ocupación, mientras que la renta se limita a la consideración de sólo tres de los estudios específicos para España, a diferencia de lo que ocurre en otros países en los que la información económica del hogar es más accesible y fiable. Únicamente 
un estudio para España, muy reciente y todavía no publicado, compara los tres factores -educación, ocupación o clase social y renta- y la evolución del gradiente desde los años noventa (Rodriguez-Caro, Vallejo-Torres y López-Valcárcel, 2016).

En los estudios para Europa, la renta familiar es uno de los indicadores clave. En 6 de los 8 estudios internacionales revisados se considera la renta familiar, y en 3 de los 8 (Devaux y Sassi, 2013; Knai et al., 2012; Bammann et al., 2013) se evalúa el impacto de los tres factores socioeconómicos.

En los estudios para niños y adolescentes la forma más frecuente de medir la posición socioeconómica es la educación de ambos padres (Costa-Font y Gil, 2013; Roskam et al., 2010). Frente a la heterogeneidad de definiciones de clase social que se encuentra en la literatura internacional, en España se suele definir según la ocupación del cabeza de familia, sola o combinada con la educación (Devaux y Sassi, 2013; Moreno et al., 2004; Costa-Font y Gil, 2013; Font-Ribera et al., 2014; Regidor et al., 2004), cuya categorización varía entre estudios. El tercer indicador de posición socioeconómica mide los ingresos del hogar (Costa-Font y Gil, 2013; Rodriguez-Caro, Vallejo-Torres y López-Valcárcel, 2016; Font-Ribera et al., 2014), en algún caso aproximados a través de la dificultad económica autopercibida (Escolar Pujolar, 2009). Con objeto de lograr comparaciones internacionales, se han definido medidas sencillas como la FAS (family affluence scale), compuesta por cuatro ítems de posesión de coche, dormitorio propio, computadora y salir de vacaciones (Duarte-Salles et al., 2011; Due et al., 2009).

La mayor parte de los estudios que consideran más de un factor de posición socioeconómica estima el efecto de cada factor por separado, aditivamente, en modelos multivariantes. Algunos estudios para adultos incorporan tanto la posición socioeconómica vivida en la infancia como la condición socioeconómica actual del individuo (Cirera et al., 2015; Regidor et al., 2004; Pérez-Hernández, 2016).

\subsection{Ajuste por otros factores de riesgo (FR) para sobrepeso/obesidad}

Todos los estudios ajustan por género y en su caso, por edad. En los estudios de niños se suele ajustar por el sobrepeso/obesidad de los padres (Knai et al., 2012; Costa-Font y Gil, 2013), es lo que se conoce como transmisión intergeneracional de la obesidad. Otros factores por los que ajustan los modelos son sedentarismo, horas de sueño, tiempo que pasan mirando televisión, publicidad, y falta de acceso a alimentos saludables, los cuales a su vez exhiben gradiente social, así como la condición de inmigrante (Font-Ribera et al., 2014).

\subsection{Medición del gradiente}

Las medidas más habituales del efecto de la condición socioeconómica (por ejemplo, el nivel educativo) sobre la obesidad son el Índice de Desigualdad 
Absoluta (IDA), que es la diferencia en prevalencia de obesidad entre los grupos educativos de mayor y menor nivel (Due et al., 2009; Devaux y Sassi, 2013) y el índice relativo de desigualdad (IRD) (Roskam et al., 2010; Due et al., 2009). Este se define como el cociente entre la categoría más alta y la más baja). Para comparaciones internacionales o entre grupos heterogéneos se estandariza por la mediana intra pais en cada categoría (Due et al., 2009). Los índices resultantes, llamados por algunos autores respectivamente Slope Index of Inequality and Relative Index of Inequality, tienen en cuenta el gradiente a lo largo de toda la distribución (Mackenbach y Kunst, 1997).

Algunos estudios emplean los índices de concentración (CI) que ajustan mejor a través de toda la distribución y conectan con la abundante literatura económica de la equidad en acceso a la atención sanitaria. El Índice de Concentración de Salud (Vallejo-Torres y Morris, 2010) mide la desigualdad en salud relacionada con los ingresos y puede ser comparada a través de los años y en diferentes regiones. El valor 0 es la perfecta igualdad, los valores positivos señalan desigualdad a favor de los ricos.

Otra medida de gradiente, más orientada a las políticas, es el Porcentaje Atribuible (PA) al nivel socioeconómico desfavorable, que por ejemplo para la educación puede interpretarse como la proporción de la obesidad asociada con un nivel educativo inferior al máximo, es decir, el que se esperaría eliminar si toda la población alcanzara el máximo nivel educativo (Gutierrez-Fisac et al., 2002). Si bien se utiliza como estimación del contrafactual de un mundo que hubiera eliminado el gradiente social (Hoffmann et al., 2015), los escenarios basados en las generalizaciones de los efectos (todo el mundo estudia en la universidad, por ejemplo), son poco realistas.

\section{4. ¿QUÉ SABEMOS DEL GRADIENTE SOCIAL DE LA OBESIDAD EN ESPAÑA Y DE SU EVOLUCIÓN?}

\subsection{Fuerte gradiente social inverso generalizado en adultos}

Aunque la prevalencia global de la obesidad parece que se va estabilizando, hay un fuerte gradiente social inverso por nivel educativo (a menor nivel educativo, mayor probabilidad de obesidad) y por situación socio-económica baja. En una comparación internacional con datos y metodología homogénea, Devaux y Sassi concluyen que España, Hungría, Reino Unido y Francia presentan el mayor gradiente educativo inverso en mujeres en términos absolutos, con IDA mayor de 10 puntos, y España, Italia y Francia tienen un IRD>4 (Devaux y Sassi, 2013; Knai et al., 2012; Hoffmann et al., 2015). También hay evidencia consistente de que las desigualdades en obesidad de origen socioeconómico persisten hasta la 
vejez. En España, las condiciones de vida en la infancia (ocupación de los padres) han dejado un rastro de grasa en las personas a lo largo de toda su vida (Cirera et al., 2015; Pérez-Hernández, 2016).

\section{2. ¿Educación, ocupación o renta? En la educación está la clave}

Un estudio comparativo internacional concluye que el gradiente por estatus socioeconómico es menor que por educación, tanto en términos absolutos como relativos, salvo en Suecia y Austria para las mujeres (Devaux y Sassi, 2013). Un estudio reciente para España estima el gradiente según renta, clase social y educación mediante el RII a lo largo de toda la distribución de IMC, utilizando regresión incondicional por cuantiles (Rodriguez-Caro, Vallejo-Torres y LópezValcárcel, 2016), y compara el gradiente desde los años noventa hasta 2014. Concluye que la educación es el factor de desigualdad de mayor impacto es decir, el gradiente educativo supera al de ingresos familiares o clase social. Este es un hecho diferencial de España respecto a otros países, sobre todo los nórdicos, en los que la renta tiene mayor protagonismo como fuente de gradiente. Así, para los trabajadores de la ciudad de Helsinki de mediana edad, la privación material en el presente predice mejor la obesidad que la educación o la ocupación (Laaksonen, Sarlio-Lähteenkorva y Lahelma, 2004).

Los adultos mayores también manifiestan un gradiente socioeconómico de los factores de riesgo cardiovascular, incluyendo la obesidad (Pérez-Hernández et al., 2016). El estudio ENRICA-Seniors ha permitido conocer el problema a finales de la década pasada (2008-2010) para la población mayor (60 años o más), según nivel socioeconómico, que se mide a través de la educación y ocupación de los individuos y también con la ocupación del padre, permitiendo atisbar transmisión intergeneracional del gradiente. Destacamos tres resultados: 1) la gran relevancia de la educación, con un Odd Ratio estimado (IC95\%) entre 0,33-0,57 para las personas sin estudios o con estudios primarios frente a los universitarios; 2) el mayor gradiente para mujeres que para hombres; 3) la ocupación del individuo tiene mayor impacto que la del padre, lo que podría interpretarse como que si bien hay cierta transmisión intergeneracional del gradiente social, que se aprecia hasta la vejez de las personas, esos condicionantes familiares se modulan con la trayectoria personal a lo largo de la vida.

\subsection{Peor para las mujeres}

Prácticamente todos los estudios para España concluyen que el gradiente es mayor en mujeres que en hombres. Un trabajo comparativo con datos de panel para nueve países europeos no encuentra gradiente inverso de renta familiar para los hombres, y únicamente se presenta gradiente para las mujeres en cinco de los 
nueve países (no en España) (Villar y Quintana-Domeque, 2009). Además, el origen de ese efecto está en que las mujeres obesas perciben rentas inferiores. Por ejemplo, entre las mujeres de Helsinki con alto nivel de ocupación y educación, la renta media de las obesas es significativamente menor que la de sus equivalentes no obesas (Sarlio-Lähteenkorva, Silventoinen y Lahelma, 2004). Esto nos lleva a la literatura sobre discriminación laboral atribuible a la obesidad, que se superpone a la discriminación por género. En España, las obesas mórbidas sufren discriminación laboral significativamente mayor que las de peso normal, particularmente si trabajan en puestos de cara al público o si viven en áreas donde la norma social sobre peso es más estricta (áreas con menor prevalencia de obesidad) (Vallejo-Torres, Morris y López-Valcárcel, 2016).

\subsection{El gradiente se mantiene estable}

La dinámica del gradiente en un país es debida a dos efectos concomitantes, la ganancia de peso a lo largo del tiempo de los individuos de la población inicial, y la incorporación de cohortes más jóvenes a la población. A nivel internacional, algunos estudios miden el aumento de peso a lo largo del tiempo con datos longitudinales de una muestra de individuos de la población inicial, otros manejan datos transversales o bases transversales repetidas, como las encuestas nacionales de salud de diferentes años.

Una comparación internacional del gradiente educativo desde 1994 hasta 2007 sugiere que en España el gradiente se ha mantenido sin cambios significativos; parece por tanto que el movimiento de la distribución poblacional de IMC ha sido homogéneo para los diferentes grupos educativos (Devaux y Sassi, 2013), igual que en Italia, Francia y el Reino Unido.

Para España no hay estudios longitudinales del gradiente que permitan una estimación fiable de su dinámica. Los estudios disponibles sugieren que el gradiente parece haber empeorado para las mujeres aunque no para los hombres entre 1987 y 1997 (Gutierrez-Fisac et al., 2002) y que se ha mantenido relativamente estable en la última década (Rodriguez-Caro, Vallejo-Torres y López-Valcárcel, 2016).

\subsection{Obesidad infantil: también hay gradiente socioeconómico}

En una revisión para España (Font-Ribera et al., 2014) de las desigualdades sociales en la salud infantil y adolescente 2000-2012, 12 de los 72 estudios analizaban el gradiente social de la obesidad, aunque solo uno (van Stralen et al., 2012) para preescolares. Esa revisión establece claramente que la educación es un factor de riesgo de gradiente mucho más influyente que los otros dos y que las desventajas de género ya son evidentes desde la infancia para las niñas, que sufren mayor gradiente que los niños. 
El estudio transversal en-KIND, con una muestra representativa de la población española entre 2 y 24 años, aportó mucha información sobre la prevalencia de obesidad y su gradiente en 1998-2000 y dio lugar a un número significativo de publicaciones relevantes (Serra-Majem et al., 2003; Aranceta et al., 2003; Roman et al., 2009). La obesidad infantil y de jóvenes (hasta 24 años) en España era mayor entre niños $(15.6 \%)$ que entre niñas $(12.0 \%)$, y mas frecuente en el intervalo de edad entre 6 y 13 años (Serra-Majem et al., 2006). Por tanto, la obesidad infantil es más prevalente en niños pero el gradiente social es mayor entre las niñas. Si la medición de las causas es difícil en los adultos, para los niños es mayor, ya que los tres factores de desigualdad -educación, renta y ocupación o clase social- se multiplican, al poder medirse para el padre, para la madre, o para ambos. El nivel educativo de la madre y el estatus socioeconómico de la familia son factores de riesgo de obesidad infantil en España que tienen la misma intensidad (el nivel inferior tiene un Odd Ratio en torno a 1.25 respecto al superior, según un modelo logístico multivariante) (Serra-Majem et al., 2006).

Un estudio para 35 países desarrollados concluye que la prevalencia de sobrepeso es mayor entre niños de familias de ingresos bajos en 21 de los 24 países de renta alta, incluido España (Due et al., 2009). También concluye este estudio que el índice relativo de desigualdad no está correlacionado con la prevalencia de sobrepeso. España ocupaba la cuarta posición de los 35 países en el ranking de sobrepeso infantil en niños, y el noveno puesto en niñas.

En España, el riesgo de sobrepeso en adolescentes, sobre todo niñas, es mucho mayor si la madre tiene nivel educativo bajo ( 27.3 de sobrepeso vs $9.6 \%$ en hijas de madres con educación superior). Ni el nivel socioeconómico de la familia ni la educación del padre son, según ese estudio, significativos (Duarte-Salles et al., 2011). En otro estudio para España (Jimenez-Pavon et al., 2010) en niños, se observó que a medida que aumenta el nivel educativo de ambos padres mejora el estado nutricional de los hijos $(\mathrm{p}<0,001)$ siendo más estrecho y significativo en el caso de las madres con alta formación académica.

No obstante, en España (2003-2006) el gradiente por renta, medido mediante curvas de concentración es realmente dramático (Costa-Font y Gil, 2013). Además, el que la madre no participe en el mercado laboral protege de riesgo de obesidad únicamente a sus niños varones, no a la niñas, según ese mismo trabajo.

\subsection{Obesidad infantil: la dinámica del gradiente}

La dinámica del gradiente de obesidad infantil en Europa, a diferencia de la de adultos, parece estar empeorando. En España, la obesidad infantil ha aumentado alarmantemente entre 2003 y 2006 (Costa-Font y Gil, 2013) así como el gradiente social, pues aumentó más entre el sector más pobre de la población, tal vez vía transmisión cultural de hábitos alimentarios aprendidos de sus padres. 


\subsection{Los mecanismos que explican el gradiente}

Los mecanismos que explican el gradiente son múltiples, y abarcan la menor receptividad a mensajes y recomendaciones de prevención primaria (Kark y Rasmussen, 2005), los patrones de alimentación menos saludable entre los niños y adolescentes de condición socioeconómica baja (Knai et al., 2012), y el entorno obesogénico o de bajo nivel socioeconómico (Moraeus et al., 2012). El fuerte gradiente educativo de los padres encontrado en prácticamente todos los estudios de sobrepeso infantil puede explicarse porque los hijos de padres mas educados ven menos la TV, están menos tiempo en su cuarto, hacen mas deporte y comen regularmente mas frutas y verduras. La obesidad de los adultos, incluso mayores, puede estar asociada a la condición socioeconómica del padre en la infancia (Cirera et al., 2015; Pérez-Hernández et al., 2016). Los hijos de padres obesos tienen una probabilidad de sobrepeso/obesidad mayor. La condición de normopeso de los padres, incluso si su condición socioeconómica es baja, protege contra el desarrollo de obesidad en los hijos, por lo que la transmisión intergeneracional de la obesidad de padres a hijos podría ser más determinante que la condición socioeconómica.

Una pregunta relevante es si las sociedades económica y socialmente mas desiguales tienden a mayor prevalencia de sobrepeso infantil. Un estudio comparativo para 11 países europeos (Knai et al., 2012) contesta afirmativamente. España está entre los tres países más desiguales económicamente (índice de Gini más alto) y entre los de mayor prevalencia de sobrepeso infantil, tanto auto reportado como medido, en los años 2000.

En un estudio comparativo de prevalencia de obesidad infantil en ocho regiones europeas (la muestra española se obtuvo en centros escolares de Zaragoza y Huesca), con metodología estandarizada, se encontró que el gradiente socioeconómico está correlacionado con la renta regional y el índice de desarrollo humano específico del país (Bammann et al., 2013).

\section{DISCUSIÓN}

\subsection{Discusión general}

La obesidad como fenómeno de masa es relativamente reciente. Sus causas son múltiples, y tiene connotaciones filosóficas sobre la libertad, el papel y responsabilidad del Estado, los ciudadanos y las empresas. De hecho, en España la estrategia NAOS para afrontar la obesidad se basa en el autocontrol y colaboración voluntaria de las empresas, sobre todo del sector de alimentación.

En España ha habido un esfuerzo importante, y despliegue de medios, para investigar el problema con fuentes primarias, desde el estudio enKid, que desveló 
la situación de los niños y jóvenes a principios de los 2000, hasta el SEEO'97 para adultos entre 25 y 60 años o el más reciente estudio ENRICA-Seniors, para los mayores de 60 años. Gracias a esos y a otros estudios podemos aproximarnos hoy al problema con más fiabilidad, y conocemos la existencia y persistencia de un gradiente social y económico de la obesidad y el sobrepeso.

Ese gradiente tiene un reflejo inmediato en términos de desigualdades de salud y en mortalidad. Una cohorte de 36.296 adultos seguida desde los años noventa (1992-96) durante una media de 12 años (EPIC-Spain) ha permitido asociar la aparición de diabetes tipo II, y sus factores causales inmediatos como la obesidad, con la situación socioeconómica relativa de la persona a lo largo de toda su vida (Cirera et al., 2015). Esta se midió como un constructo que combina la ocupación del padre durante la infancia y el nivel educativo alcanzado por el individuo. El estar en la categoría socioeconómica inferior representa un aumento de riesgo de diabetes tipo 2, comparada con la categoría superior, del 90\% para los hombres y del $126 \%$ para las mujeres.

Las desigualdades socioeconómicas en obesidad se traducen en desigualdades sociales en mortalidad. A partir de los datos de mortalidad atribuible a la obesidad para 21 poblaciones se ha estimado (Hoffmann et al., 2015) el impacto que tendría la eliminación del gradiente educativo de la obesidad: si se eliminaran las diferencias en obesidad entre grupos educativos, se reduciría la desigualdad relativa en mortalidad por todas la causas entre las personas con baja y con alta educación hasta en un $12 \%$ para los hombres y $42 \%$ para las mujeres. Las poblaciones del estudio incluyen muestras de Madrid, Barcelona y País Vasco, y en los tres casos el gradiente educativo de la mortalidad femenina atribuible a la obesidad es notoriamente mayor que para la mayor parte de las poblaciones del estudio. No obstante, a este tipo de ejercicios basados en el método del upward-levelling scenario, que consisten en suponer que se consigue que todos estudien, les falta realismo y sobre todo no responden a la pregunta esencial: ¿cómo se consigue que todos los ciudadanos asciendan al nivel educativo superior?

La posición socioeconómica se suele medir mediante educación, clase social ocupacional, e ingresos, pero son escasos los estudios que permiten comparar el efecto de cada uno de esos factores por separado, y hay que avanzar sobre los modelos conceptuales, contrastables empíricamente, acerca de cómo estas tres características interactúan entre sí y con los cambios en el IMC, de forma dinámica. Por ejemplo, el graduarse en la escuela secundaria pasó de ser un paso hacia los estudios universitarios a convertirse una condición necesaria para ingresar al mercado laboral. Con todo, en España parece claro que el nivel educativo es la dimensión más asociada con el gradiente de obesidad y sobrepeso.

Por otra parte, la medida del gradiente social se puede hacer en términos absolutos o relativos, y las implicaciones no son las mismas. Algunos trabajos muestran cambios en el gradiente absoluto pero estabilidad temporal en el relativo. 


\subsection{Posibles sesgos en los estudios}

La principal limitación de la mayor parte de los estudios es el carácter observacional y transversal de los datos, que permiten establecer correlaciones pero no causaciones. En algunos casos, los datos proceden de encuestas nacionales o regionales de salud representativas de la correspondiente población y no se han diseñado específicamente para el estudio. Cuando se quieren trazar tendencias temporales se utilizan cortes transversales repetidos (por ejemplo, uniendo varios años de la Encuesta Nacional de Salud). Esos datos no permiten tener en cuenta la causalidad inversa (de la posición socioeconómica hacia el IMC), la obesidad como mediadora o concurrente con otras causas ni los posibles sesgos de selección, por atrición y clasificación entre otros. En suma, la evidencia disponible es fundamentalmente observacional, y la mayor parte de los estudios establecen correlación más que causalidad. Son pocos los que se basan en diseños potencialmente capaces de superar esa limitación.

Hay dos fuentes preocupantes de sesgos: los errores de medida (autodeclaración de peso y talla) y el sesgo de selección por no participación. Los cuestionarios con medidas autodeclaradas conducen por lo general a una subestimación del verdadero valor del IMC, dando lugar a un sesgo de clasificación de las personas en las colas de las distribuciones y a una considerable subestimación de la prevalencia de sobrepeso/obesidad. Esos errores de clasificación, que podrían estar diferenciados por género, se superponen a los sesgos inducidos por el efecto cohorte (por ejemplo, tener estudios secundarios completos no es lo mismo hoy que hace 30 años). En suma, el sesgo de autodeclaración de peso y talla podría resultar en un sesgo de infraestimación del gradiente social.

Por otra parte, si la falta de participación es mas frecuente en personas obesas de bajo estatus socioeconómico, se podría producir sesgo en los resultados, igual que si los obesos abandonaran más la escuela en los años mas recientes.

Son necesarios más estudios con alta calidad metodológica con diseño prospectivo a largo plazo.

\section{CONCLUSIÓN. EL PAPEL DE LAS POLÍTICAS PARA REDUCIR EL GRADIENTE}

Las políticas que buscan reducir el gradiente no necesariamente coinciden con las que intentan reducir la prevalencia de la obesidad general. Por ejemplo, en un reciente estudio para España (Ventosa y Urbanos-Garrido, 2016) con datos de la Encuesta Nacional de Salud 2011-12, se concluye que las intervenciones que tratan de reducir el sedentarismo asociado a la actividad principal, aunque posiblemente reduzcan o prevengan la obesidad, amplificarían su gradiente 
social. En este trabajo hemos mostrado que el gradiente se encuentra desde la primera infancia y persiste hasta edades avanzadas, que la obesidad se transmite entre generaciones, y la condición socioeconómica desfavorable marca un riesgo añadido de sufrir sobrepeso y obesidad a lo largo de la vida. La transmisión intergeneracional de la (mala) salud en España, por la doble vía de la renta o posición socioeconómica de los padres, y de la educación, impone restricciones a la movilidad social (Pinilla, López-Valcárcel y Urbanos-Garrido, 2016). La obesidad es un buen ejemplo.

Las políticas educativas y de protección económica de la familia dirigidas a hogares de condición socioeconómica baja serán efectivas no solamente en reducir las desigualdades de renta, también en último término contribuirán a reducir desigualdades en salud. Asimismo, la práctica de la discriminación positiva a personas y hogares desfavorecidos, tanto en los ámbitos educativo como en la promoción de la actividad física en el tiempo libre puede contribuir a prevenir la obesidad y a reducir las desigualdades en salud (Ventosa y Urbanos-Garrido, 2016).

La obesidad es un problema de salud que añade gran carga de enfermedad a las sociedades desarrolladas y en desarrollo, y su evolución epidémica preocupa a las autoridades sanitarias. La obesidad refleja un problema de desigualdad social que no parece que se esté reduciendo. Hoy en día, es a la vez causa y efecto de desigualdades socioeconómicas, de modo que las políticas contra la obesidad son, en último término, no solamente políticas de salud sino también de equidad. Además, hay diferencias sustanciales por género en la relación entre obesidad y condición socioeconómica. El gradiente es mayor para las mujeres. Por tanto, las políticas anti obesidad son también políticas en pro de la igualdad de género.

Las políticas educativa y laboral pueden contribuir a reducir el problema. Este planteamiento concuerda con el más general de "Salud en Todas las Políticas", pues la solución a las desigualdades socioeconómicas relacionadas, como causa y como efecto, con la obesidad, se encuentra mayormente fuera del sector sanitario, en el universo de las empresas y en el de las políticas sociales.

\section{BIBLIOGRAFÍA}

ARANCETA, J., et al. (2001), "Influence of sociodemographic factors in the prevalence of obesity in Spain. The SEEDO'97 Study”, European Journal of Clinical Nutrition, vol. 55, núm. 6, pp. 430-435.

ARANCETA, J., et al. (2003), "Sociodemographic and lifestyle determinants of food patterns in Spanish children and adolescents: the enKid study", European Journal of Clinical Nutrition, núm. 57, pp. S40-S44. 
BAMMANN, K., et al. (2013), "Socioeconomic factors and childhood overweight in Europe: results from the multi-centre IDEFICS study", Pediatric Obesity, vol. 8, núm. 1, pp. 1-12.

BANERJEE, A. V. y DUFLO, E. (2009), "The Experimental Approach to Development Economics", Annual Review of Economics, vol. 1, núm. 1, pp. 151-178.

BIBILONI M., et al. (2010), "Prevalence and risk factors for obesity in Balearic Islands adolescents", British Journal of Nutrition, vol. 103, núm. 1, p. 99-106.

BLACK, J. L. y MACINKO, J. (2008), "Neighborhoods and obesity", Nutritional Review, vol. 66, núm. 1, pp. 2-20.

CHRISTAKIS, N. A. y FOWLER, J. H. (2007), "The spread of obesity in a large social network over 32 years", New England Journal of Medicine, vol. 357, núm. 4, pp. 370-379.

CIRERA, L., et al. (2015), "Life-course social position, obesity and diabetes risk in the EPIC-Spain Cohort", The European Journal of Public Health, p. ckv218.

COLE, T. J., et al. (2000), "Establishing a standard definition for child overweight and obesity worldwide: international survey", BMJ, núm. 320(7244), p. 1240.

COSTA-FONT, J. y GIL, J. (2013), "Intergenerational and socioeconomic gradients of child obesity", Social Science Eु Medicine, núm. 93, pp. 29-37.

DEVAUX, M., et al. (2011), "Exploring the relationship between education and obesity, OECD Journal: Economic Studies, vol. 1, pp. 121-159.

DEVAUX, M. y SASSI, F. (2013), "Social inequalities in obesity and overweight in 11 OECD countries", European Journal of Public Health, vol. 23, núm. 3, pp. 464-469.

DUARTE-SALLES, T., et al. (2011), "Social inequalities in health among adolescents in a large southern European city", Journal of Epidemiology Community Health, vol. 65, núm. 2, pp. 166-173.

DUE, P., et al. (2009), "Socioeconomic position, macroeconomic environment and overweight among adolescents in 35 countries", International Journal of Obesity, vol. 33, núm. 10, pp. 1084-1093.

ESCOLAR PUJOLAR, A. (2009), "Social determinants vs. lifestyle in type 2 diabetes mellitus in Andalusia (Spain): difficulty in making ends meet or obesity?", Gaceta Sanitaria, vol. 23, núm. 5, pp. 427-432.

FONT-RIBERA, L., et al. (2014), "The study of social inequalities in child and adolescent health in Spain", Gaceta Sanitaria, vol. 28, núm. 4, pp. 316-325.

GARCINUNO, A. C., et al. (2010), "Social disparities in the prevalence of overweight and obesity in adolescents", Anales de Pediatría, vol. 73, núm. 5, pp. 241-248. 
GEYER, S., et al. (2006), "Education, income, and occupational class cannot be used interchangeably in social epidemiology. Empirical evidence against a common practice", Journal of Epidemiology and Community Health, núm. 60(9), pp. 804-810.

GONZALEZ JIMENEZ, E., et al. (2012), "Influence of family environment of the development of obesity and overweight in a population of school children in Granada (Spain)”, Nutrición Hospitalaria, vol. 27, núm. 1, pp. 177-184.

GUTIERREZ-FISAC, J., et al. (2002), "The size of obesity differences associated with educational level in Spain, 1987 and 1995/97", Journal of Epidemiology and Community Health, vol. 56, núm. 6, pp. 457-460.

HILLIER-BROWN, F. C., et al. (2014), "A systematic review of the effectiveness of individual, community and societal level interventions at reducing socioeconomic inequalities in obesity amongst children”, BMC Public Health, núm. 14, p. 834.

HOFFMANN, R., et al. (2015), "Obesity and the potential reduction of social inequalities in mortality: evidence from 21 European populations", The European Journal of Public Health, p. ckv090.

JIMENEZ-PAVON, D., et al. (2010), "Influence of socioeconomic factors on fitness and fatness in Spanish adolescents: the AVENA study", International Journal of Pediatry Obesity, vol. 5, núm. 6, pp. 467-73.

KARK, M. y RASMUSSEN, F. (2005), "Growing social inequalities in the occurrence of overweight and obesity among young men in Sweden", Scandinavian Journal of Public Health, vol. 33, núm. 6, pp. 472-477.

KAUTIAINEN, S., et al. (2009), "Sociodemographic factors and a secular trend of adolescent overweight in Finland", International Journal Pediatric Obesity, vol. 4, núm. 4, pp. 360-370.

KNAI, C., et al. (2012), "Socioeconomic patterning of childhood overweight status in Europe", International Journal of Enviromental Research and Public Health, vol. 9, núm. 4, pp. 1472-1489.

LAAKSONEN, M., SARLIO-LÄHTEENKORVA, S. y Lahelma, E. (2004), "Multiple dimensions of socioeconomic position and obesity among employees: The Helsinki Health Study”, Obesity Research, vol. 12, núm. 11, pp. 1851-1858.

LARRANAGA, N., et al. (2007), "Prevalence of obesity in 4-18-year-old population in the Basque Country, Spain", Obesity Reviews, vol. 8, núm. 4, pp. 281-287.

LOBSTEIN, T., MILLSTONE, E. y PORGROW RESEARCH, T. (2007), "Context for the PorGrow study: Europe's obesity crisis", Obesity Reviews, núm. 8 Supl. 2, pp. 7-16. 
MACKENBACH, J. P. y KUNST, A. E. (1997), "Measuring the magnitude of socio-economic inequalities in health: an overview of available measures illustrated with two examples from Europe", Social Science $\mathbb{E}$ Medicine, vol. 44, núm. 6, pp. 757-771.

MAGNUSSON, M., et al. (2014), "Social Inequalities in Obesity Persist in the Nordic Region Despite Its Relative Affluence and Equity", Current Obesity Reports, núm. 3, pp. 1-15.

MARMOT, M., et al. (2008), "Closing the gap in a generation: health equity through action on the social determinants of health", The Lancet, núm. 372(9650), pp. 1661-1669.

MOLARIUS, A., et al. (2000), "Educational level, relative body weight, and changes in their association over 10 years: an international perspective from the WHO MONICA Project", American Journal of Public Health, vol. 90, núm. 8, pp. 1260-1268.

MORAEUS, L., et al. (2012), "Multi-level influences on childhood obesity in Sweden: societal factors, parental determinants and child's lifestyle", International Journal of Obesity, vol. 36, núm. 7, pp. 969-976.

MORENO, L. A., et al. (2004), "Micro-environmental and socio-demographic determinants of childhood obesity", International Journal of Obesisty and Related Metabolic Disordes, núm. 28 Sup. 3, pp. S16-20.

PAMPEL, F. C., KRUEGER, P. M. y DENNEY, J. T. (2010), "Socioeconomic disparities in health behaviors", Annual Review of Sociology, núm. 36, pp. 349-370.

PÉREZ-HERNÁNDEZ, B., et al. (2016), "Desigualdades sociales en los factores de riesgo cardiovascular de los adultos mayores de España: estudio ENRICA-Seniors", Revista Española de Cardiología.

PINILLA, J. G., LÓPEZ-VALCÁRCEL, B. y URBANOS-GARRIDO, R. (2016), "Estimating direct effects of parental occupation on Spaniards' health by birth cohort", BMC Public Health, (en prensa).

REGIDOR, E., et al. (2004), "Obesity and socioeconomic position measured at three stages of the life course in the elderly", European Journal Clinical Nutrition, vol. 58, núm. 3, pp. 488-494.

RODRÍGUEZ-CARO, A., VALLEJO-TORRES, L. y LÓPEZ-VALCÁRCEL, B. (2016), "Unconditional quantile regressions to determine the social gradient of obesity in Spain 1993-2014", International Journal for Equity in Health, vol. 15, núm. 1.

ROMAN, B., et al. (2009), "Physical activity in children and youth in Spain: future actions for obesity prevention", Nutrition Reviews, num. 67, sup. 1, pp. S94-S98.

ROSKAM, A. J., et al. (2010), "Comparative appraisal of educational inequalities in overweight and obesity among adults in 19 European countries", International Journal of Epidemiology, vol. 39, núm. 2, pp. 392-404. 
SARLIO-LÄHTEENKORVA, S., SILVENTOINEN, K. y LAHELMA, E. (2004), "Relative weight and income at different levels of socioeconomic status", American Journal of Public Health, vol. 94, núm. 3, pp. 468-472.

SASSI, F. y ORGANISATION FOR ECONOMIC CO-OPERATION AND DEVELOPMENT (2010), Obesity and the economics of prevention fit not fat, OECD, Paris.

SERRA-MAJEM, L., et al. (2006), "Prevalence and deteminants of obesity in Spanish children and young people", British Journal of Nutrition, núm. 96, Sup. 1, pp. S67-72.

SERRA-MAJEM, L., et al. (2003), "Obesidad infantil y juvenil en España. Resultados del Estudio enKid (1998-2000)”, Medicina clínica, núm. 121(19), pp. 725-732.

SOBRADILlO, B., AGUIRRE, A. y ARESTI, U. (2004), Curvas y Tablas de Crecimiento (Estudios longitudinal y transversal), Instituto de Investigación sobre Crecimiento y Desarrollo Fundación F. Orbegozo Eizaguirre, Bilbao.

SORIGUER, F., et al. (2004), "Prevalence of obesity in south-east Spain and its relation with social and health factors", European Journal of Epidemiology, vol. 19, núm. 1, pp. 33-40.

SWEETING, H. N. (2007), "Measurement and definitions of obesity in childhood and adolescence: a field guide for the uninitiated", Nutrition Journal, vol. 6, num. 1, p. 1.

VALLEJO-TORRES, L. y MORRIS, S. (2010), "The contribution of smoking and obesity to income-related inequalities in health in England", Social Science Ë Medicine, vol. 71, núm. 6, pp. 1189-1198.

VALLEJO-TORRES, L., MORRIS, S. y LÓPEZ-VALCÁRCEL, B. (2016), "Obesity and perceived work discrimination in Spain", International Journal for Equity in Health, vol. 15, núm. 1.

VAN STRALEN, M. M., et al. (2012), "Weight status of European preschool children and associations with family demographics and energy balancerelated behaviours: a pooled analysis of six European studies", Obesity Reviews, núm. 13, Sup. 1, pp. 29-41.

VENTOSA, M. M., y URBANOS-GARRIDO, R. M. (2016), "Disentangling effects of socioeconomic status on obesity: a cross-sectional study of the Spanish adult population" Economics $\mathcal{E}$ Human Biology, vol. 22, pp. 216224.

VILLAR, J. G. y QUINTANA-DOMEQUE, C. (2009), "Income and body mass index in Europe”, Economics $\mathcal{G}$ Human Biology, vol. 7, núm. 1, pp. 73-83. 
El gradiente social de la obesidad en España. ¿Qué sabemos y Qué deberíamos saber?

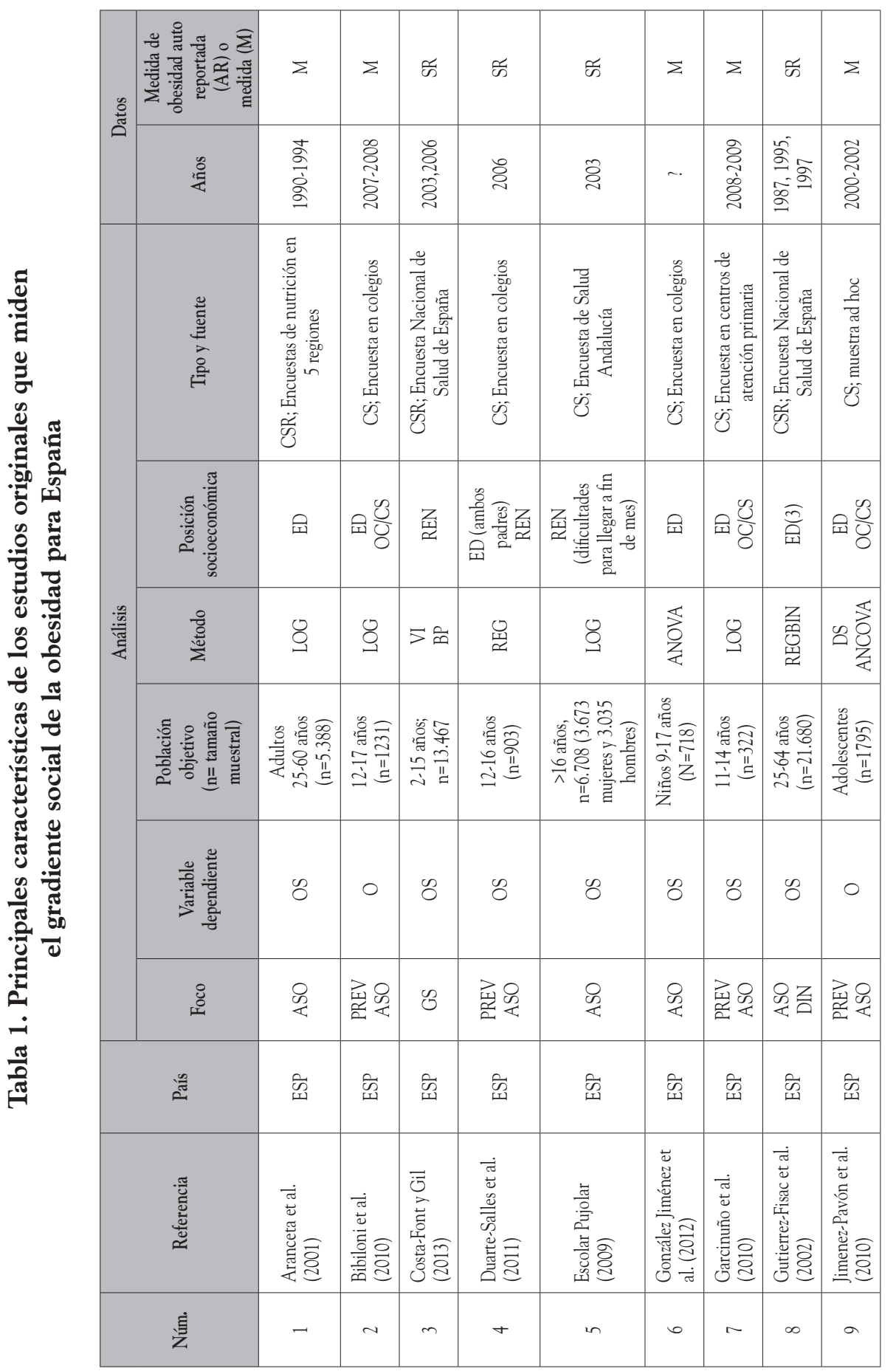

icade. Revista cuatrimestral de las Facultades de Derecho y Ciencias Económicas y Empresariales, no 99 septiembre-diciembre 2016, ISSN: 1889-7045 
Josefina Panetta y Beatriz G. López-Valcárcel

\begin{tabular}{|c|c|c|c|c|c|c|c|c|c|}
\hline 总 & 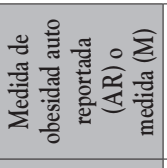 & $\Sigma$ & $\Sigma$ & $\Sigma$ & $\Sigma$ & $\Sigma$ & $\Sigma$ & $\Sigma$ & 囷 \\
\hline & 题 & 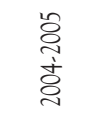 & 家 & $\begin{array}{l}\overrightarrow{8} \\
\grave{\delta} \\
\grave{\delta}\end{array}$ & 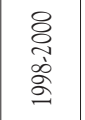 & $\because$ & $\begin{array}{l}\text { 우 } \\
\text { ᄋे̀ }\end{array}$ & 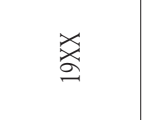 & 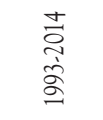 \\
\hline \multirow{6}{*}{ 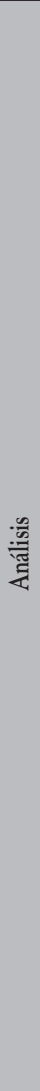 } & 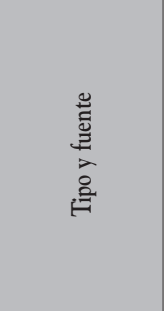 & 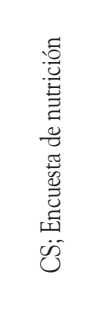 & 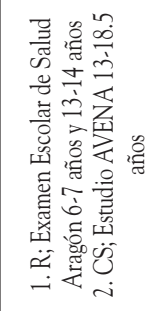 & 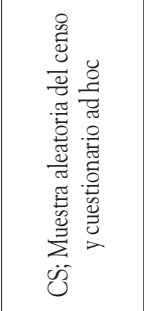 & 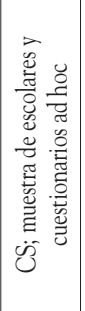 & 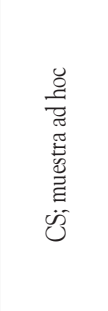 & 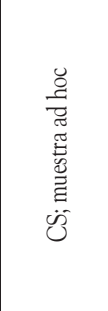 & ż & 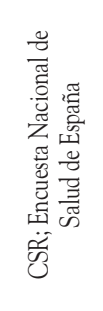 \\
\hline & 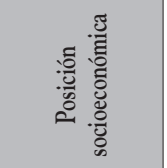 & 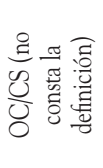 & 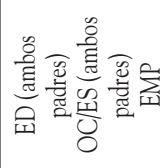 & 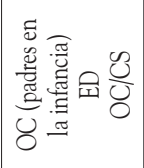 & 오응 & 㝇 & 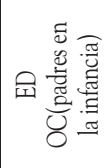 & 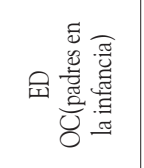 & 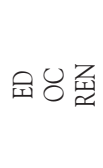 \\
\hline & 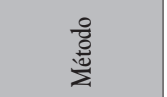 & О & 8 & 岃 & 8 & 8 & & & $\mathscr{q}$ \\
\hline & 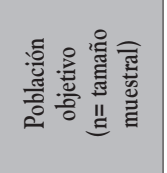 & 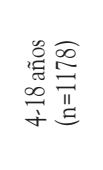 & 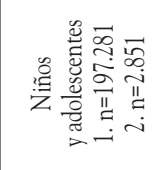 & 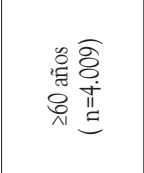 & 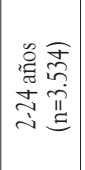 & 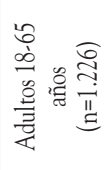 & 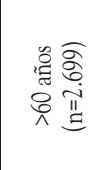 & 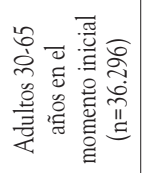 & 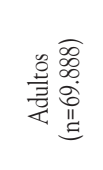 \\
\hline & 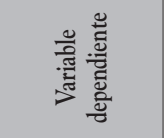 & क & क & क & क & 0 & 0 & 0 & m \\
\hline & 董 & 㱏安 & 㚣苔 & 足 & 密号 & 嵒足 & \& & 足 & 足台 \\
\hline & 沓 & 戹 & 离 & 畐 & 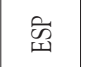 & 密 & ज्ञ & 点 & 穹 \\
\hline & 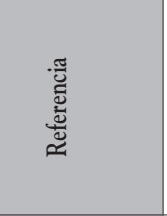 & 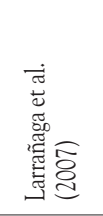 & 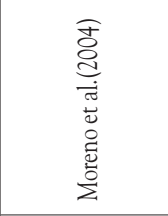 & 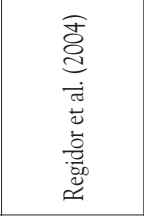 & 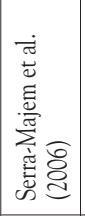 & 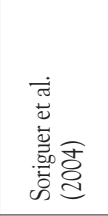 & 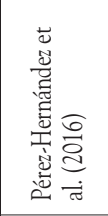 & 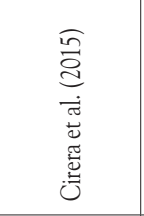 & 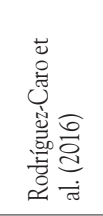 \\
\hline & 豈 & 으 & $=$ & $\simeq$ & $\approx$ & \pm & $\simeq$ & 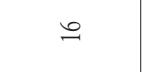 & $\simeq$ \\
\hline
\end{tabular}


El GRAdiente SOCial de la obesidad en España. ¿Qué SAbemos y Qué Deberíamos saber?

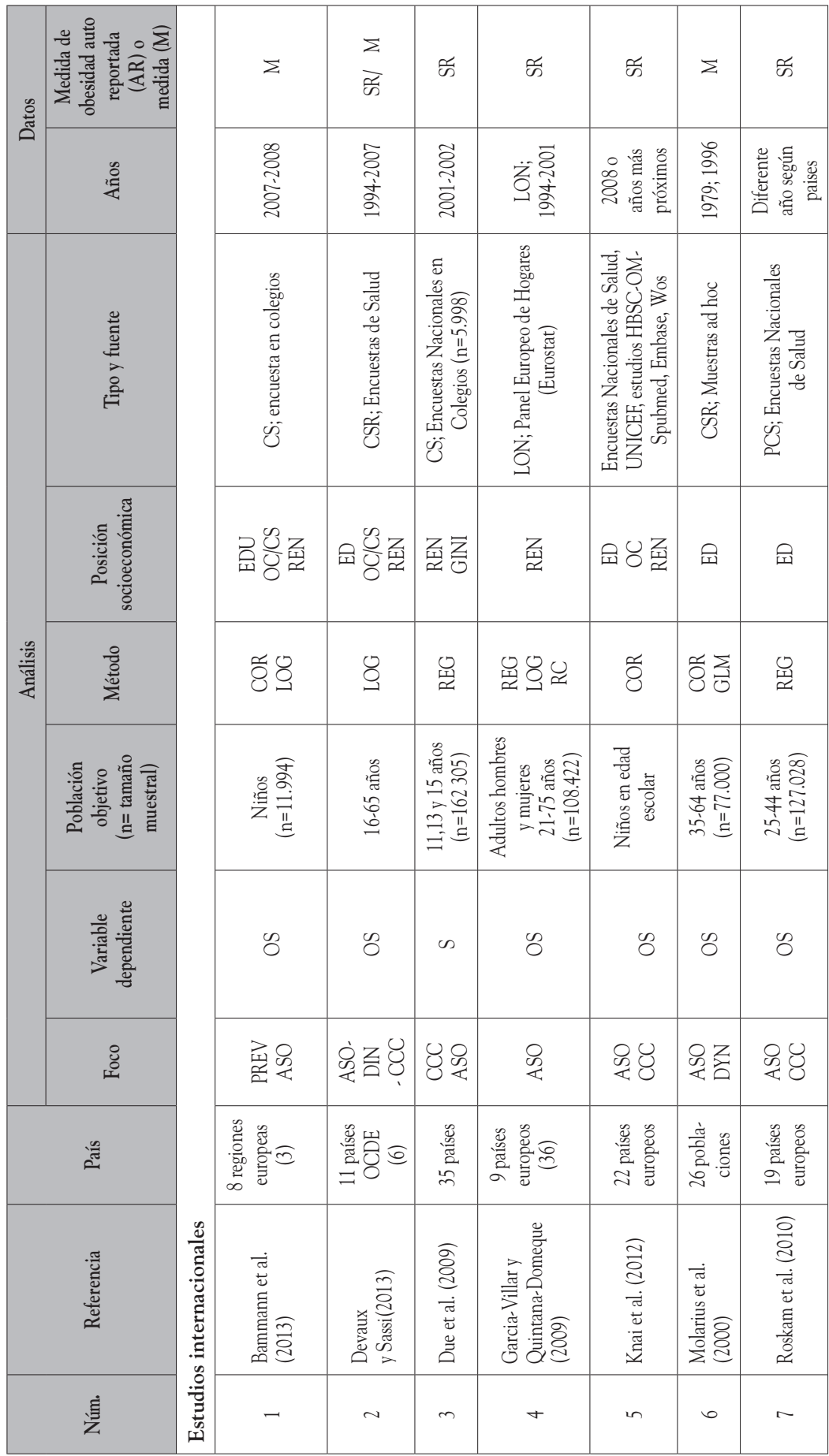

icade. Revista cuatrimestral de las Facultades de Derecho y Ciencias Económicas y Empresariales, no 99 septiembre-diciembre 2016, ISSN: 1889-7045 
Josefina Panetta y Beatriz G. López-Valcárcel

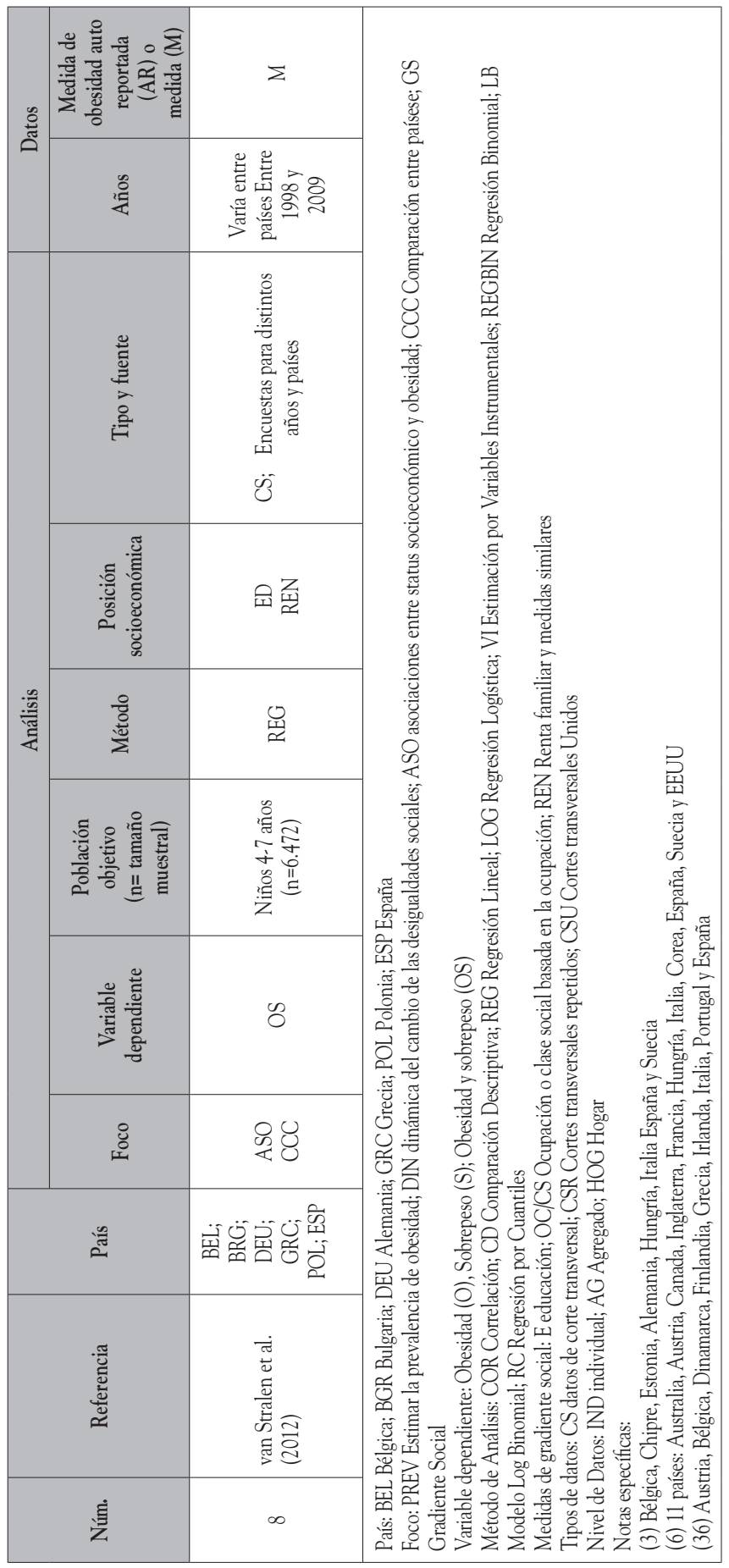

